FOURIER DECOMPOSITION PARAMETERS FOR THE HALO RR LYRAE VARIABLES U AND V CAELI

L. Hansen and J.O. Petersen

Copenhagen University Observatory, Øster Voldgade 3

DK-1350 Copenhagen K, Denmark

Abstract. UBVRI light curves are obtained for the two halo

RR Lyrae variables $U$ Caeli with period 0.420 days (73

observations) and $V$ Caeli with period 0.571 days ( 42

observations). It is shown that their light curve

characteristics are very similar to those of field RR Lyrae

stars.

Fourier decompositions are studied for all five

magnitudes and the resulting amplitude ratios and phase

differences are discussed. The differences in the Fourier

decomposition parameters between the five magnitudes are

shown to be relatively small. Comparisons of the Fourier

decomposition parameters for the two halo RR Lyrae stars

with recently published data for field RR Lyrae stars show

no systematic differences.

INTRODUCTION

With the purpose of studying bump progression sequences and other properties of Cepheid type variables we obtained UBVRI photometry of the Population II Cepheid KZ Centauri with period $1.52 \mathrm{~d}$ and used Fourier decomposition technique for analysis of these observations (Petersen \& Hansen, 1984) and of mean light curves for RR Lyrae stars in the globular cluster $\omega$ Centauri (Petersen, 1984).

In the present study we investigate two halo RR Lyrae stars for which no accurate photometry has been available until now. The main purpose is to derive the Fourier decomposition parameters and compare amplitude ratios and phase differences calculated for U, B, V, R, and I.

THE OBSERVATIONS OF U AND $V$ CAELI

The UBVRI photometry was obtained during six nights from November 29th to December 5th, 1982, by means of the Danish $1.5 \mathrm{~m}$ telescope at La Silla. Both the observations and the data reductions were performed by the methods described in Petersen \& Hansen (1984).

LIGHT AND COLOUR CURVES

Fig. 1 presents the light and colour curves of $\mathrm{V}$ Caeli. It is seen that the light curves in all bands are remarkably similar. To a good approximation they are simply scaled versions of one another, just as we found for $\mathrm{KZ}$ Centauri. The $\mathrm{U}$ and $\mathrm{B}$ curves are virtually identical, the only systematic variation in U-B being a small decrease close to light maximum followed by a similar increase. In these respects $U$ Cae is very similar to $\mathrm{V}$ Cae.

In $\mathrm{V}$ Cae a gradual change in light curve form from $\mathrm{U}$ to $\mathrm{I}$ is indicated in the phase interval $f=0.70-0.90$ : While the $U$ curve may have a small positive slope, the B curve seems practically horizontal and the $V, R$, and I curves show negative, gradually decreasing slopes. 
Figure 1. Light and colour curves of $\mathrm{V}$ Caeli. Eighth order Fourier fits are shown in all cases and individual observations are given except for $R$, $U$, and $R-I$.

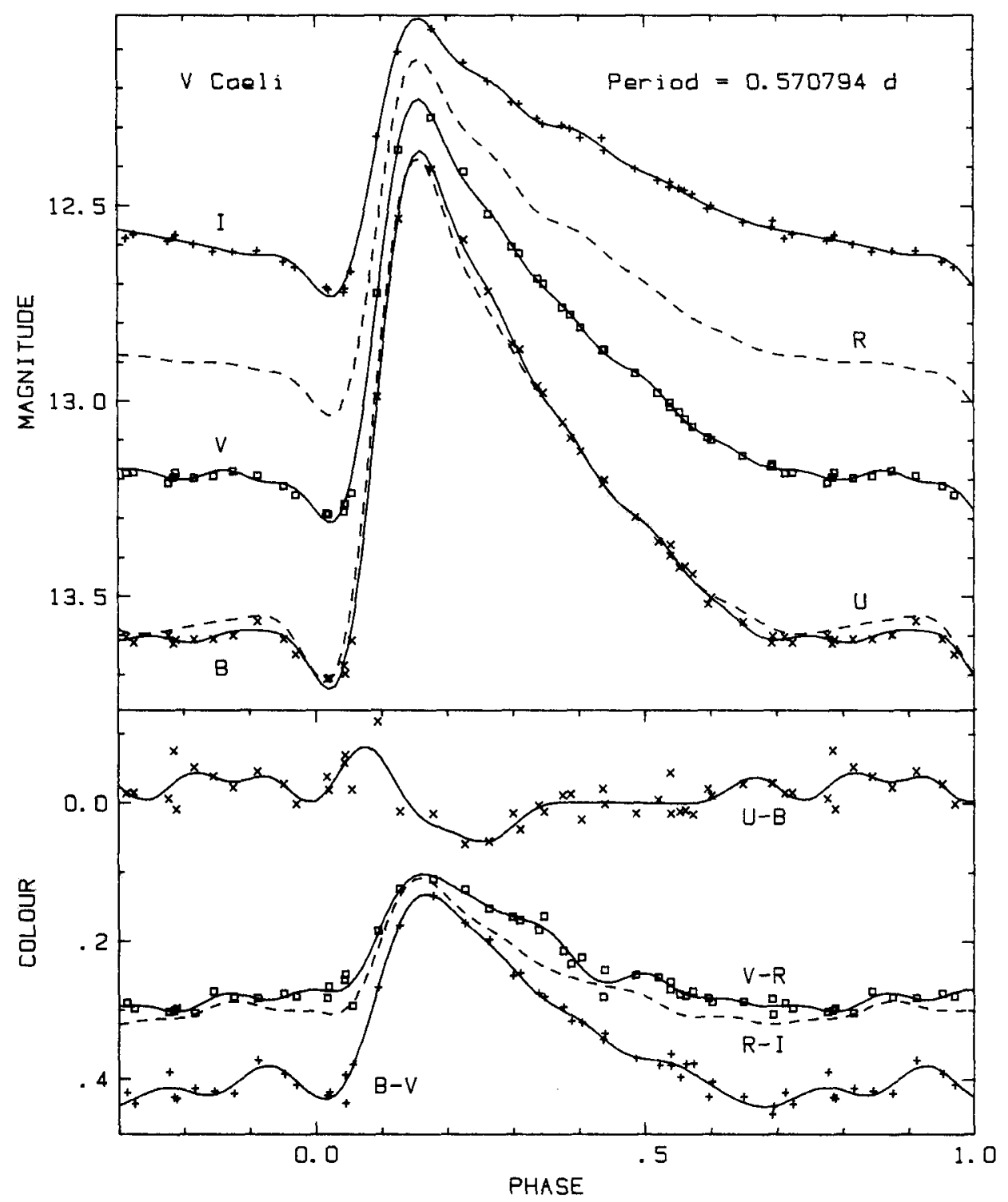


In the following we also find small systematic changes in the Fourier decomposition parameters.

Photographic light curves for $U$ and $V$ Cae have been published by Hoffmeister (1956). For U Cae Hoffmeister gives the light variation $\mathrm{m}_{p h}=11.6-13.1$ with a scatter of about \pm 0.2 . This agrees well with our result $B=11.4-13.0$. Hoffmeister remarks that the large scatter he found present around light minimum is probably real. our observations (six nights) show a scatter somewhat larger than expected from the photometric accuracy, but smaller than about \pm 0.04 . We cannot decide whether this scatter is due to a non-repeating light curve, a small period change or somewhat unfavourable observing conditions. In order to study this problem we plan to observe $U$ and $V$ Cae again at a later date. For $\mathrm{V}$ Cae Hoffmeister gives $m_{p h}=11.9-13.1$ while we find $\mathrm{B}=12.3-13.7$. Due to the large scatter in the photographic light curves no detailed comparison of light curve features can be performed.

Comparing our observations of $U$ and $V$ Cae with the very detailed light curves for field RR Lyrae stars, published by Lub (1977), in the period interval $0.42-0.57 \mathrm{~d}$, we note a nice qualitative agreement in light curve features. In particular the small dip occuring in $U$ and $V$ Cae just before the steep rise to maximum also occurs in most of Lub's curves.

\section{FOURIER DECOMPOSITIONS}

Table 1 gives the results of 8 th order Fourier decompositions of the light curves of $\mathrm{U}$ and $\mathrm{V}$ Caeli calculated by the methods described in Petersen \& Hansen (1984). The values given for the $V$ magnitude should correspond to the data given by Simon \& Teays (1982) for RR Lyrae field stars. A comparison of the phase differences $\varphi_{21}$ and $\varphi_{31}$ and the

Table 1. Amplitude ratios, $R_{k l}$, and phase differences $\varphi_{k l}$, for $U$ and $V$ Caeli calculated for decompositions of order $N=8$. For $V$ magnitudes these quantities are given directly. In other cases we give $\Delta_{k l}$ defined as the value for the magnitude in question minus the corresponding value for the $V$ magnitude.

\begin{tabular}{|c|c|c|c|c|c|c|c|}
\hline \multirow{2}{*}{ STAR } & \multirow{2}{*}{ MAG } & $\mathrm{R}_{\mathrm{kl}}$ & or & $\Delta_{\mathrm{kl}}$ & $\varphi_{\mathrm{kI}}$ & or & $\Delta_{\mathrm{kl}}$ \\
\hline & & 21 & 31 & 41 & 21 & 31 & 41 \\
\hline \multirow[t]{2}{*}{ U Cae } & V & .50 & .36 & .22 & 3.78 & 7.95 & 5.88 \\
\hline & $\begin{array}{l}\mathrm{U} \\
\mathrm{B} \\
\mathrm{R} \\
\mathrm{I} \\
\end{array}$ & $\begin{array}{r}-.01 \\
-.02 \\
.01 \\
.01 \\
\end{array}$ & $\begin{array}{r}.00 \\
-.02 \\
.01 \\
.02 \\
\end{array}$ & $\begin{array}{r}.00 \\
-.01 \\
.01 \\
.01 \\
\end{array}$ & $\begin{array}{r}-.08 \\
-.06 \\
.11 \\
.26 \\
\end{array}$ & $\begin{array}{r}-.14 \\
-.12 \\
.18 \\
.43 \\
\end{array}$ & $\begin{array}{r}-.27 \\
-.16 \\
.21 \\
.63 \\
\end{array}$ \\
\hline \multirow[t]{2}{*}{ V Cae } & $\mathrm{V}$ & .47 & .34 & .22 & 3.87 & 8.06 & 6.02 \\
\hline & $\begin{array}{l}\mathrm{U} \\
\mathrm{B} \\
\mathrm{R} \\
\mathrm{I} \\
\end{array}$ & $\begin{array}{r}-.02 \\
-.01 \\
.01 \\
.01 \\
\end{array}$ & $\begin{array}{l}.00 \\
.00 \\
.03 \\
.02 \\
\end{array}$ & $\begin{array}{r}.01 \\
-.01 \\
.03 \\
.03 \\
\end{array}$ & $\begin{array}{r}-.13 \\
-.11 \\
.11 \\
.33 \\
\end{array}$ & $\begin{array}{r}-.18 \\
-.18 \\
.22 \\
.66 \\
\end{array}$ & $\begin{array}{r}-.19 \\
-.22 \\
.22 \\
.70 \\
\end{array}$ \\
\hline
\end{tabular}


amplitude ratio $R_{21}$ by means of Simon \& Teays' figures show perfectly normal values for $U$ and $V$ Caeli.

Table 1 shows that the differences in Fourier decomposition parameters calculated for different photometric systems are relatively small in particular for the amplitude ratios. Comparison of the data for $\mathrm{U}$ Cae with those for $\mathrm{V}$ Cae show a good agreement. In all cases the differences have same sign, but the $U$ Cae data have on the average somewhat smaller numerical values.

Similar data have been obtained for the Population II

Cepheid KZ Centauri with period 1.52 d by Petersen \& Hansen (1984). Due to the conspicuous differences in light curve characteristics between $\mathrm{KZ}$ Cen and the RR Lyrae stars, it is not surprising that we find quite different values of $R_{k I}$ and $\varphi_{k I}$. But it is interesting that the differences in $R_{k J}$. and $\varphi_{k l}$ between the values determined for the $\mathrm{V}$ magnitude and the other bands are very similar. For $R_{k l}$ these differences are very small, and for $\varphi_{k l}$ we find in all cases same sign and numerical values that are only slightly larger on the average for $\mathrm{KZ}$ Cen than for the RR Lyrae stars.

Presupposing that photographic magnitudes correspond to $B$, we can for RR Lyrae stars estimate the differences in Fourier decomposition parameters expected between $\mathrm{m}_{p h}$ and $\mathrm{V}$ curves by the values given for $B$ in Table 1 . It is seen that these differences are only 0.00 to -0.02 for $R_{k 1}$ and -0.10 to -0.15 for $\varphi_{21}$ and $\varphi_{31}$. As remarked in Petersen (1984) uncertainties of this order of magnitude are not important in discussions of bump progression sequences.

REFERENCES

Hoffmeister, C. (1956). Veröi ntlichungen Sonneberg, Band 3, 5. Lub, J. (1977). Astron. Astrophys. Suppl., $29,345$.

Petersen, J.O. (1984). Astron Astrophys., submitted.

Petersen, J.O. \& Hansen, L. (1984). Astron. Astrophys. , in press.

Simon, N.R. \& Teays, T.J. (1982). Astrophys. J., 261, 586. 